\title{
A NEW FORMULA FOR THE BERNOULLI NUMBERS ${ }^{1}$
}

\section{H. L. GARABEDIAN}

It is the purpose of this note to exhibit a unique method for deriving what appears to be a new formula for the Bernoulli numbers. We obtain the formula

or

$$
\begin{aligned}
B_{k+1}=\frac{(-1)^{k+1}(k+1)}{2^{k+1}-1} & \sum_{i=0}^{k} \frac{\Delta^{i} a_{0}}{2^{i+1}}, \\
a_{n}=(1+n)^{k}, k=0,1,2, \cdots, & ,
\end{aligned}
$$

$$
\begin{array}{r}
B_{k+1}=\frac{(-1)^{k+1}(k+1)}{2^{k+1}-1}\left[\frac{1}{2}+\frac{1}{2^{2}}\left(1-2^{k}\right)+\frac{1}{2^{3}}\left(1-2 \cdot 2^{k}+3^{k}\right)+\cdots\right. \\
\left.+\frac{1}{2^{k+1}}\left\{1-C_{k, 1^{k}} 2^{k}+C_{k, 2} 3^{k}-+\cdots+(-1)^{k} C_{k, k}(k+1)^{k}\right\}\right], \\
k=0,1,2, \cdots .
\end{array}
$$

Some of the Bernoulli numbers computed from (1) are $B_{1}=-1 / 2$, $B_{2}=1 / 6, B_{3}=0, B_{4}=-1 / 30, B_{5}=0, B_{6}=1 / 42, B_{7}=0, B_{8}=-1 / 30$, $B_{9}=0, B_{10}=5 / 66$. Evidently, the known formulas ${ }^{2}$ which give the Bernoulli numbers in explicit form, in comparison with formula (1), yield only the numerical values of these numbers with even indices. While none of the formulas under discussion could possibly serve a useful purpose in computing unknown numbers with high indices, formula (1) appears to be the simplest in form and the one best adapted to computation of numbers with low indices.

Our method of obtaining (1) consists in summing the divergent series

(2) $\quad \sum_{n=0}^{\infty}(-1)^{n}(n+1)^{k}=1-2^{k}+3^{k}-+\cdots, \quad k=0,1,2, \cdots$,

using two consistent methods of summation, one of which assigns a value to (2) which involves the Bernoulli numbers. Equating the two values thus obtained we get the desired formula.

First, we sum the series (2) by the method of Abel. To this end we

\footnotetext{
1 Presented to the Society, December 29, 1939.

${ }^{2}$ See, for example, Niels Nielsen, Traité Élémentaire des Nombres de Bernoulli, 1923.
} 
have to make some preliminary observations. The Bernoulli numbers may be defined by the relation ${ }^{3}$

$$
\frac{x}{e^{x}-1}=B_{0}+\frac{B_{1}}{1 !} x+\frac{B_{2}}{2 !} x^{2}+\cdots,
$$

which is valid at least for small values of $x$. Now, consider the series

$$
f(t)=e^{-t}-e^{-2 t}+e^{-3 t}-+\cdots,
$$

which converges for $e^{-t}<1$ and hence for $t>0$. Moreover, we can write

$$
\begin{aligned}
f(t) & =\frac{e^{-t}}{1+e^{-t}}=\frac{1}{e^{t}+1}=\frac{e^{t}+1-2}{e^{2 t}-1} \\
& =\frac{1}{e^{t}-1}-\frac{2}{e^{2 t}-1}=\frac{1}{t} \frac{t}{e^{t}-1}-\frac{1}{t} \frac{2 t}{e^{2 t}-1} .
\end{aligned}
$$

We may now expand the last expression in (4) using (3). We obtain for small values of $t>0$ the relation

$$
\begin{aligned}
f(t)=\frac{1}{t} & {\left[B_{0}+\frac{B_{1}}{1 !} t+\frac{B_{2}}{2 !} t^{2}+\cdots\right] } \\
& -\frac{1}{t}\left[B_{0}+\frac{2 B_{1}}{1 !} t+\frac{2^{2} B_{2}}{2 !} t^{2}+\cdots\right],
\end{aligned}
$$

or

$$
\sum_{n=0}^{\infty}(-1)^{n} e^{-(n+1) t}=-\sum_{n=0}^{\infty} \frac{2^{n+1}-1}{(n+1) !} B_{n+1} t^{n} .
$$

Differentiating $k$ times with respect to $t$, we have

$$
\begin{aligned}
\sum_{n=0}^{\infty}(-1)^{n}(n+1)^{k} e^{-(n+1) t} & \\
& =(-1)^{k+1} \sum_{n=k}^{\infty} \frac{2^{n+1}-1}{(n+1) !} B_{n+1} \cdot n(n-1) \cdots(n-k+1) t^{n-k} .
\end{aligned}
$$

Now, allowing $t$ to approach zero through positive values, we obtain

$$
\lim _{t \rightarrow 0^{+}} \sum_{n=0}^{\infty}(-1)^{n}(n+1)^{k} e^{-(n+1) t}=(-1)^{k+1} \frac{2^{k+1}-1}{k+1} B_{k+1} .
$$

Putting $x=e^{-t}$ in the series on the left in (5), we have a power series

${ }^{3}$ Euler, Institutiones Calculi Differentialis . . , vol. 2, 1755, §122. 
with radius 1 . Thus, the statement (5) implies that the series (2) is summable by the method of Abel (or is summable A) to the value given in (5). ${ }^{4}$

Next, we sum the series (2) by the Cesàro method. We need the following theorem. ${ }^{5}$

Theorem. The series $\sum_{n=0}^{\infty}(-1)^{n} a_{n}$ is exactly summable $(C, \nu)$, $(\nu=1,2,3, \cdots)$, to the value

$$
\sum_{n=0}^{\nu-1} \frac{\Delta^{n} a_{0}}{2^{n+1}}
$$

provided that

$$
\Delta^{i} a_{0}=0, \quad \Delta^{\nu-1} a_{0} \neq 0, \quad i \geqq \nu \geqq 1 .
$$

Since the general term in the series (2) is a polynomial of degree $k$, its differences of order higher than $k$ all vanish. Thus, due to our theorem, the series (2) is summable $(C, k+1)$ to the value

$$
\sum_{n=0}^{k} \frac{\Delta^{n} a_{0}}{2^{n+1}}, \quad a_{n}=(n+1)^{k}, k=0,1,2, \cdots .
$$

Now, it is well known that Abel summability includes Cesàro summability of positive integral orders. Then, the series (2) must be summable $A$ to the value (6). Equating (5) and (6) we get the desired formula:

$$
B_{k+1}=\frac{(-1)^{k+1}(k+1)}{2^{k+1}-1} \sum_{i=0}^{k} \frac{\Delta^{i} a_{0}}{2^{i+1}}, \quad a_{n}=(n+1)^{k}, k=0,1,2, \cdots .
$$

\section{NORTHWESTERN UNIVERSITY}

${ }^{4} \mathrm{~K}$. Knopp, Theorie und Anwendung der unendlichen Reihen, 1922, 2d edition, 1924, p. 508.

${ }^{5}$ H. L. Garabedian, this Bulletin, vol. 45 (1939), pp. 592-596. 